\title{
Urgences
}

\section{Saut de note}

\section{Pour une déontologie de la pensée pure}

\section{Robert Hébert}

Numéro 31, mars 1991

Poétique de la note

URI : https://id.erudit.org/iderudit/025632ar

DOI : https://doi.org/10.7202/025632ar

Aller au sommaire du numéro

Éditeur(s)

Urgences

ISSN

0226-9554 (imprimé)

1927-3924 (numérique)

Découvrir la revue

Citer cet article

Hébert, R. (1991). Saut de note : pour une déontologie de la pensée pure.

Urgences, (31), 7-18. https://doi.org/10.7202/025632ar

Ce document est protégé par la loi sur le droit d'auteur. L'utilisation des services d'Érudit (y compris la reproduction) est assujettie à sa politique d'utilisation que vous pouvez consulter en ligne.

https://apropos.erudit.org/fr/usagers/politique-dutilisation/
Cet article est diffusé et préservé par Érudit.

Érudit est un consortium interuniversitaire sans but lucratif composé de l'Université de Montréal, l'Université Laval et l'Université du Québec à Montréal. Il a pour mission la promotion et la valorisation de la recherche. https://www.erudit.org/fr/ 


\section{Saut de note Pour une déontologie de la pensée pure}

Robert Hébert

C'est l'endroit que je trouve le plus propice pour finir mes livres, à Montréal, à cause de la neige. Qui rend tout silencieux. Et c'est blanc. (Voir la signification du blanc comme source de tous les possibles dans la 8 Genèse.) C'est à la fois le blanc de l'écriture, la page blanche et le silence. Un silence ouaté...

Michel Serres à M. Pelletier, Le Devoir, 20 mars 1982.

Grosso modo, la note infrapaginale ou déportée à la fin des chapitres ou du livre a deux fonctions par rapport au Texte. Tout d'abord, permettre à l'auteur de donner ses références et ses sources, jouer de la citation démonstrative, épingler l'état bibliographique d'une question. Ensuite, expliciter ou expliquer, gloser sur quelques difficultés ou éclaircies, entretenir un rapport privilégié avec le lecteur. Dans l'univers du savoir et de la doxa, une note interagit toujours entre le connu de l'auteur et l'inconnu du lecteur. Rebaptisons cela sans trop appuyer. La première fonction concerne la note autoritaire que j'appelle " tournée vers le dedans du Texte »; il y a des "autorités", un continuum de connaissances déjà là, un savoir microcosmique qui font être tel nouveau texte. À la limite de l'honnêteté intellectuelle que chacun souhaite voir chez autrui, la clôture de cette note est le pédantisme, le marketing d'un curriculum vitæ. Et rien de plus éprouvant, entre autres notes incontrôlables, pisse-vinaigre, fantômes..., que les brefs renvois sans citations, où le lecteur ne sait jamais si l'occasion de l'appel d'une note (expression, thème, idée...) ne masque pas beaucoup de guillemets dans le texte. Ainsi la philosophie professionnelle des revues et périodiques devient souvent le long recopiage de ses lettres de créance. Qui écrit à partir de qui, pour faire quoi ? La seconde fonction concerne la note dialoguale que j'appelle "tournée vers le dehors du Texte"; notes personnelles et baroques, proleptiques, tiroirs d'exemples...; j'inclus également la note précautionneuse qui consiste en bas de page à affaiblir les 
8

arguments d'en haut, bémoliser ses affirmations. Langue fourchue. À la limite de la logorrhée centrifuge à quoi le plaisir de l'explication échappe difficilement, la clôture de cette note est d'autres formes de pédantisme ou la digression qui charrie. Mais parfois la perche dialoguale de la note vers le dehors joue de toute évidence avec les rêvasseries de ce qui fut un jour les autorités du dedans. Belles bouteilles à la mer. Par exemple, entre lourdes citations et adresses au lecteur, on sait que les très longues notes $f$ ), i), j) et $p$ ) du second Discours sur l'origine de l'inégalité parmi les hommes de Rousseau sont fondamentales dans l'histoire des idées. De Chateaubriand, Tocqueville, Lovejoy, Chinard à Lévi-Strauss beaucoup se sont lancés sur les traces de Jean-Jacques, son orang-outang, ses lectures américano-canadiennes. Même s'il n'y accordait pas d'importance, lui pour qui les notes étaient l'occasion de "s'amuser une seconde fois à battre les buissons" avec les bâtons rompus de l'auteur.

Synthèse pointilleuse des pratiques de lecture et d'écriture, synthèse du pouvoir-dire et du vouloir-dire ou de leur suspension, la note participe avant tout de la rhétorique typographique de la mise en page. Comment se présente une note? Regardez le blanc longitudinal au bas de la page à quoi s'adossent des caractères plus petits ou alors le blanc zébré d'une autre page qui rassemble les notes: ce geste assez banal force à affronter une dimension décisive de la pensée. Une note se détache, fait bouger le blanc des orbites, la tête, les mains comme si on suivait une partie de volley-ball entre l'équipe championne et quelques ichno-secrétaires devant le filet séparateur - , fait sauter le texte (à poursuivre) pour mieux le rattraper. Dimension décisive d'un sursaut dans la surprise des rapports entre le texte souverain et autre chose. Trahirais-je ici un secret? Ceux et celles qui font de la recherche jusqu'à la limite de la saturation, qui sont à l'affût d'un ton nouveau savent que la confrontation fondamentale du savoir avec «lui-même" réside au fond dans la note: réseau territorial des sources, trafic ou non des connaissances, aveux parfois bouleversants, censures obliques, passion du risque, guerre des consciences qui se démarque du propos central. Nota bene: une petite note peut faire crouler, s'écrouler un empire d'illusions tenaces. Alors qu'appelle-t-on dire ou ne pas dire ailleurs autre chose? Dans tous les cas, la pratique de la note, c'est le walkie-talkie sous la superstructure solennelle du Texte, c'est le stylodrome de la pensée pure. Ca fait courir hors texte, ça met l'Inconscient sur des pistes, ça use les Adidas de l'hyperconscient lecteur. 
La doctrine standard de la note repose sur l'utilité, la modération et la santé oculaire du lecteur... Sans trop s'aventurer sur la quantification de ses usages - où et quand doiton faire un appel de note? question délirante mais radicale à poser, ligne après ligne - , on aboutit souvent à une sorte de notologie négative; ne faites pas ceci, évitez cela:

II ne faut rien écrire dans les notes qui soit plus important que le texte ou qui lui soit contraire; les notes ne sont pas des catacombes destinées à recevoir tout le système de fiches de l'auteur. Les notes ne sont pas destinées à corriger les oublis du texte; on ne les écrit pas quand l'ouvrage est écrit... 1

Cependant, ce n'est pas le moindre paradoxe que les grands érudits, les grands penseurs et/ou grosses vedettes, ces Géants dont on ne rêve même plus de taper l'épaule, qui font progresser le savoir, les idées, les néo-frissons seconds, sont précisément ceux et celles qui dérogent à la doctrine standard. Ou bien excès de scholarship notarial, genre bibliographie des sources rédigée dans un code pour extra-terrestre ou alors excès de silence, le rien angélo-diaphane ou presque rien sous la brique (souvent appuyé par une haine de la note qui est en fait une haine de l'histoire). Doublement fascinants pour des raisons complémentaires. Moi, pour oublier la logorrhée philosophique un peu plate et prévisible, j’aime les notes compactes, prédicatives, bourrées de titres inter/nationaux, exubérance des périodiques, toponymes, dates comme si le texte à lire tremblait de son passé présent ${ }^{2}$, me faisant

1 G. Yelle, Travail scientifique en disciplines ecclésiastiques, Montréal, Grand Séminaire de Montréal, 1945, p. 104. La métaphore des catacombes est un peu l'équivalent clérical de celle de dépotoir. Plus joyeux entre grammaires typographiques et manuels de style, cf. Mary-Claire van Leunen, $A$ Handbook for Scholars, New York, Alfred A. Knopf, 1978: “ By using footnotes judiciously you can fill your reader in on general information he lacks, satisfy his curiosity about fine points, whisper delicious tidbits in his ears, and share with him an occasional small frolic... Avoid the cultivation of a schizophenic style. The author of the text and the author of the footnotes should be recognizably the same person... Neither the lackluster text nor the frenzied footnote is a goal to strive after ", p. 91-92, 101-102. Dans un autre couloir, davantage littéraire, Gérard Genette, Seuils, coll. "Poétique", Paris, Seuil, 1987, "Les notes", p. 293-315.

2 Creative scholarship et histoire des idées à l'anglo-américaine sont particulièrement stimulantes. Entre autres ouvrages emblematiques, lire Thornas J. Schlereth, The Cosmopolitain Ideal in Enlightenment Thought..., Notre Dame, Univ. of Notre Dame Press, 1977. Trente pages de notes, précédées d'un essai bibliographique (p. 137-179) qui situe le topos. Dans les marges, j'ai ajouté quelques notes et remarques sur les rapports canadiens des trois sujets de l'ouvrage: Franklin, Hume et Voltaire. 
penser mon propre tremblement lorsque je prétends moimême à la démiurgie de mon espace-temps; mais j'aime aussi certains poèmes fraternels de E.E. Cummings ou Guillevic sur un large blanc... La paix souveraine! Si la note marque le continuum lectoriel entre le passé de l'auteur et l'avenir du lecteur, si sur chaque page imprimée ou dans chaque livre un espace de notes est toujours pensable (que j'appelle le cadastre pointilleux), visible ou invisible, alors la question devient celle-ci: COMMENT ANNOTER SES PROPRES PENSEEES OU ALORS, POURQUOI NE PAS LE FAIRE? Cette question est essentiellement déontologique. Je ne veux pas ici théoriser des procédés ou faire des procès d'intention, les limites de la conscience artisanale m'intéressent davantage. Je laisserai cette question en suspens, avec son aura d'humour, abrupt, à la Ding et Dong. Le problème philosophique de la note, c'est l'histoire millénaire de l'annotation sur papyrus et codices de parchemin, c'est aussi la pratique créatrice de la non-note! À la fois dans leurs conditions de possibilité et d'impossibilité.

Ouvrons Le grand Robert à l'entrée lexicale «note». Celleci repose sur une division entre le champ musical, harmonique, olfactif même, et le champ graphique depuis le XVII siècle, jusqu'aux acceptions plus récentes, le compte à payer et l'évaluation chiffrée (" avoir de bonnes notes en philo»); on pourrait sans doute ici filer les métaphores dans l'espace du discours philosophique, montrer que la Transcendance est la note de fond du parfum textuel, enfin... je laisse à d'autres le soin de nous édifier sans nous éditer... Pour revenir à la boîte à lunch, les citations 16 et 23 (Flaubert), 19 (Alain) et 26 (Valéry) sont au cœur des diverses problématiques. Écoutons spécialement l'hygiéniste Alain: "Toujours revenir aux grands textes; n'en point vouloir d'extraits... Et je dis aussi à l'œuvre sans notes. La note, c'est le médiocre qui s'accroche au beau. L'humanité secoue cette vermine. "Va pour la première partie de la citation, d'autant plus que le mode contemporain de l'intertexte, citations et clichés, prélèvements et triturations sont souvent reliés à des lectures incomplètes, paquetées, bombes à retardement qui reviennent parfois chez l'auteur grâce à un lecteur-recenseur qu'il n'avait pas prévu. Quant à la deuxième partie de la citation, le problème se corse. Qu'est que l'CEuvre dans le sens achevé, non contaminé du terme? De toute évidence, la note ici concerne les annotations critiques d'une main étrangère. Annulée précisément, toute l'histoire des moyens techniques et des formes culturelles grâce auxquels nous croyons commenter des œuvres 
pures $^{3}$. Histoire datée depuis Alexandrie, III $-\mathrm{II}{ }^{\mathrm{e}}$ siècles avant notre ère: rassemblement des supports scripturaux, établissement de textes (corrections, améliorations...), commentaires et scolies, code de repérage. Autrement, à travers Pyrénées et Apennins, équipes de polyglottes et chasseurs de manuscrits, moines copistes, érudits, interpolateurs qui nous font encore douter et extrapoler. On a construit Homère, Platon, Aristote, les "Écritures Saintes" et les auteurs classiques au moins jusqu'au XVI ${ }^{\mathrm{e}}$ siècle - au fil des besoins de la civilisation et de chaque renaissance -, aujourd'hui il n'est pas sûr que l'on sache comment pensaient tous nos "vestigieux" Grecs ni ce qu'ils pensaient sous l'éclair méditerranéen du mot philosophia. Corpus éternellement clos, cousu à la mitaine. Peu à peu l'invention de l'imprimerie a fait croire à l'intention originelle de l'auteur maitre-d'œuvre, sans perte aucune, mais il n'en est rien; elle a multiplié d'autres prosélytismes intéressés, d'autres contraintes sur l'écrit tout en aiguisant la conscience du sujet de l'écriture. Chapeau Montaigne! Cela, il faudrait maintenant le comprendre du point de vue d'une nouvelle vermine, moqueuse des glorioles du jour!

La page blanche de l'après-Gutenberg a surtout fait tomber le commentaire en bas de page. Et cela est la grande affaire, l'unique affaire à ruminer ici. Le texte sacré du Moyen Âge était encadré de gloses et de scolies extrêmement denses qui faisaient office de notes. Dans les marges, en manchette et même entre les lignes; bloc compact «qui a horreur du blanc ", économie oblige. Peu à peu grâce au génie des humanistes-typographes de la Renaissance et aux technologies expansives de la pulpe, la page s'aère sur le papier nouveau, démarque items et blocs significatifs, respire, clarifie visuellement idées et choses. Ensuite, l'alinéa creuse du blanc à gauche de la page, vroumm!, les notes en manchette glissent au bas de la page, woupss!! Au XVIII' siècle, c'est un fait accompli. À la lisibilité médiévale du Texte succède une autre lisibilité ${ }^{4}$ de la pensée pure. Comment et pourquoi? En

3 Ne soyons pas injustes avec Alain combattant la sclérose universitaire, luimême archi-commentateur, au point d'en faire l'homme de paille du problème la haine moderne de la note vient directement de Descartes et des premiers psycho-épistémologues britanniques (jusqu'à G. E. Moore). Étonnant phénoménologue, penseur collégial, poète (comme Platon et Nietzsche), Alain est une clé oubliée de la philosophie française: lire Histoire de mes pensées, Paris, Gallimard, 1936, sans aucune note mais entièrement imprimee en italiques.

4 Sur cette histoire matérielle de la pensée, lire L. D. Reynolds et N. G. Wilson, D'Homère à Érasme. La transmission des classiques grecs et latins, 
donnant à l'auteur la possibilité de démarquer visuellement les concaténations de sa pensée ainsi que celle d'inverserrecycler la fonction du commentaire: désormais il est libre d'annoter-commenter ou non son " propre" texte.

Quelque chose s'est donc passé dans la rationalité occidentale. L'invention typographique de la philosophie moderne - puisqu'il faut parler ici de philosophie - est une affaire de glotte, avec déplacement paginal de la glose: curieuse conjugaison d'un Je très auteur, fascination de la Science et haine de l'Histoire (et donc de la note scolaire). Exemples parlants entre tous: le Discours de la méthode (1637) de Descartes est le premier ouvrage de philosophie écrit en français, publié en paragraphes! Et la sixième "partie" est une quasi-note digressive sur le nouveau contrat public de l'auteur. Dans les Méditations (1641), pas une note, aucune citation mais, coup de génie, "Objections » et réponses-explications notoires de Descartes sont rejetées, enclavées dans l'épaisseur du livre; et ce qui pour nous serait note dialoguale (précautionneuse) apparaît comme lettre-préface « $\grave{A}$ Messieurs les doyens et docteurs... " Lisez donc à voix haute cette prose, style serpent qui s'apparente un peu au genre dévotionnel. Difficile à maintenir plus de dix minutes. Le vortex des conjonctions, locutions conjonctives (le fameux "car, encore que») et adverbes est hallucinant, surtout en début de paragraphe... Révolutionnaire, le philosophe moderne se fonde, argumentecommente son cheminement hyperbolique vers la vérité.

L'histoire de la philosophie après le classique René, c'est la démultiplication de l'entre-glose des egos, c'est aussi le développement fou du livre en tant que support matériel de la "pensée»: le "paratexte" (dédicaces, épigraphes, préfaces, remerciements, titres et intertitres, notes, appendices...), le

\footnotetext{
Paris, CNRS, 1984. II n'est pas impossible que nos philologues maniaques donnent la main aux nouveaux bibliologues, textologues, editeurs littéraires, anthropologues et sémiologues de la literacy, penseurs de l'oral et de l'imprimé. Sur la question du blanc et de la mise en page, je m'inspire des deux contributions de Roger Laufer dans la monumentale Histoire de l'édition française, sous la dir. de Henri-Jean Martin et Roger Chartier, Paris, Promodis, 1982-1984: t. I, "L'espace visuel du livre ancien ", p. 479-497, et t. II, "Les espaces du livre ", p. 128-139. Du même, "Du ponctuel au scriptural... ", Langue française, Paris, $n^{\circ} 45$, février 1980 , numéro passionnant sur la ponctuation. II est dommage que le livre (puissant par ses bornes paradoxales) de Marshall McLuhan, La galaxie Gutenberg. Genèse de I'homme typographique (1962, traduit pour la francophonie par Jean Paré, médité par Umberto Eco) n'ait pas produit de créneau herméneutique parmi nos philosophes universitaires.
} 
dispositif typo-éditorial (pages de couverture, format, numérotation de paragraphes, signes de ponctuation, italiques, diagrammes et schémas, sommaires, index...) et la répartition des blancs selon les genres (maximes, aphorismes, dialogues, traités...). Sans oublier la foi expérimentale ou le flair intéressé des imprimeurs-éditeurs. Toute pensée pure signale son matériau et s'«approfondit" avec l'évolution de ces procédés. Notification généralisée! Voyez les alinéas très secs chez Hobbes, souvent exhaustifs dans leur fonction définitionnelle, quasi épigrammatique; Spinoza herméneute réintègre, sous un apparent more geometrico appendice, scolies et explications monstrueuses (à faire rugir de haine ou de plaisir rabbins ou libertins), beaucoup plus importantes que ses C.Q.F.D.; blancheur et souveraineté encrée de la maxime chez La Rochefoucauld, Leibniz commentateur du tic au tac avec Locke dans cette partie de volley-ball posthume qu'est Nouveaux essais... (déjà le dialogue continental-analytique), Bayle encastrant ses notes et remarques très hardies dans son Dictionnaire historique et critique, Diderot, joyeux entrepreneur de l'alphabet encyclopédique, qui réinvente aussi la typographie du dialogue. Le XVIII ${ }^{\mathrm{e}}$ siècle jouera gaiement de tous les apostrophes et clins d'œil ichnographiques: remarquable mise au dehors du dedans bibliographique des Lumières.

Au sens originel du terme, c'est-à-dire médiéval conservé en droit -, le Texte s'oppose aux commentaires et aux notes. Bien, mais à quel Texte revenir aujourd'hui? Platon dialogique? Spinoza? Hegel? Wittgenstein gnomique? Au propre Glas de Derrida avec ses manchettes in et sa compacité très médiévale? Resacralisation biblique sous le couvert d'une parodie des procédés. Il faut sentir que toutes les réponses à cette question un peu absurde sont réellement absurdes: pour des raisons à la fois éditoriale et théorétique. (Il n'y a pas d'édition "définitive" de quelque écrit que ce soit et sauter vers autre chose demeure notre lot.) Par ailleurs, bloc-notes, marginalia, esquisses composent souvent le nouveau texte de l'auteur, nouvelle piste de travail pour la critique génétique ${ }^{5}$; mais comment penser l'arbitraire

5 Dans le domaine littéraire, voir l'approche de Louis Hay, " Le texte n'existe pas ". Réflexions sur la critique génétique ", Poétique, Paris, no 61, février 1985, p. 147-158. D'une façon générale, l'avant-texte des CEuvres (notes de lectures, journaux, carnets d'observations... en rapport méme aux premiers brouillons) a toujours fourni des matériaux aux interprétations nouvelles. L'édition annotée des Notebooks du poète et philosophe Coleridge par la canadienne Kathleen 
14

du texte définitif? Et surtout son équivalent en philosophie? Une certitude demeure: il y aura toujours plus d'écriture que d'écrits et plus de commentaires que de textes. Nous saisissons à peine les conséquences épistémologiques de cette Loi: béance et "zigonnage" des mécanismes créateurs, lancée des possibles interprétations, néanmoins hypostase des écritures saintes de chaque génération. Revenons donc à notre question de déontologie: COMMENT ANNOTER SES PROPRES PENSÉES OU ALORS, POURQUOI NE PAS LE FAIRE? Si la note infrapaginale représente une sorte de crise typographique qui pointe à la fois vers l'origine "autoritaire" et la finalité "dialoguale" d'une pensée, met à nu le stylodrome de chacun et chacune devant le cadastre pointilleux de son propre Occident, visible ou invisible, cette question devient ravageuse, sauvage dans sa naïveté. Feuilletons quelques grands monuments significatifs du XIX siècle. Siècle besogneux qui a expérimenté jusqu'au bout, avant le $\mathrm{XX}^{\mathrm{e}}$ siècle, la volonté de tout savoir ou alors, de ne plus rien savoir pour avoir trop compris.

Voilà! pas une note infrapaginale dans La phénoménologie de l'esprit (1807) de Hegel - l'édition de Berlin 1841 inscrit trois références abrégées -, publié en caractère gothique, vestige très territorial par rapport à la révolution du romain et de l'italique; compact teuton, sans intertitres (ajoutés un siècle plus tard par Lasson) et dont la clausule est une adaptation d'un vers de l'innommé Schiller: "Du calice de ce royaume des esprits/s'écoule jusqu'à lui sa propre infinité, " Happy end ou punch rétrospectif? Dieu seul le sait. Observez cependant Herr Doktor Hegel devant sa bibliothèque personnelle; chaque figure de la Phéno implique une pile de livres appuyée contre une autre, manceuvres esclaves à disposer, traduire et diriger sous la baguette rieuse de la sursomption. Et voici les longues digressions anti-hégéliennes, à la fois acrobatiques et "pognées" de Johannes Climacus alias Kierkegaard dans son Post-scriptum... aux miettes philosophiques (1846); avec comme sous-titre «Composition mimico-pathético-dialectique, apport existentiel». Peut-on imaginer la rage déconstructive de Soeren, perdu quelque part dans la platitude danoise? Et puis, à l'ombre du noumène kantien interprété-habillé par l'Écossais Carlyle, le

Coburn, depuis 1957, est une grande aventure historique et trans-atlantique; on lira sa propre autobiographie, In Pursuit of Coleridge, Toronto-London, The Bodley Head, 1977. 
puissant Moby Dick (1851) de Melville ouvert par un répertoire étymologique, jet de citations autoritaires et les deux notes troublantes au chapitre 42 sur la blancheur (hideuse) du cachalot. Toujours le blanc... La première édition de ce beau poème biologique intitulé The Origin of Species (1859) ne contient aucune note ni référence précise; l'expression "survival of the fittest" emprunté par Darwin à Spencer viendra avec la main des critiques et un affaiblissement de sa propre argumentation, bien que la sixième édition incorporera dans le texte additions et corrections. Bref, les parasites sont une propriété des corps textuels vivants. Â l'opposé de la procédure, Le capital de Marx (1867, année du Pacte fédératif). Superbe fête de la note autoritaire et dialoguale: 110 pages de pointilleuses notes dans l'édition Garnier-Flammarion (1969), $1 / 6^{\mathbf{e}}$ du propos théorique. Merveilles de wit, de sarcasmes, d'ironie, d'informations journalistiques, scrapbook de lucidité et de révolte. Oui, allez lire le commentaire que Karl fait à partir de cette observation: "Les Esquimaux de l'Est léchaient les articles qu'on leur vendait à mesure qu'ils les recevaient" (ch. III, note 2). Enfin, il est curieux de remarquer que La naissance de la tragédie (1871), coup d'envoi du jeune philologue Nietzsche formé dans les poussières notariales de Bâle, ne comporte pas une note; vingt-cinq paragraphes numérotés; à partir du seizième, Nietzsche commence à parler aux Allemands. Je m'arrête ici parce que Nietzsche fera plus tard sauter l'idiosyncrasie de la «philosophie allemande", vertu dormitive pour beaucoup. Mais aussi toute innocence philosophique à venir. Â force de décoder le cadastre pointilleux du quelconque Texte, il devient le penseur des guillemets et non plus de la note ${ }^{6}$. Crucifié entre l'origine assignable de toute pensée et des gloses impossibles. Descartes avait subverti la glose scolastique sous ses cogitations paragraphées; Nietzsche intègre son instinct philologue à la pratique du guillemet ironique, dans la forme fragment. À la fin, restera de l'auteur un pur dehors dévissé de son passé, déraison typographique qui aura simplement retrouvé quelques notes improvisées sur un vieux piano. Ainsi s'entend la fin d'une certaine philosophie européenne.

6 Voir Éric Blondel, "Les guillemets de Nietzsche: philologie et généalogie ", dans Nietzsche aujound'hui? 2. Passion, coll. « 10/18", Paris, UGÉ, 1973, p. 153-178, sans oublier Heinz Wismann, "Nietzsche et la philologie", loc. cit., p. 325-335. Le retour français à Nietzsche vers la fin des années 1960 (et son acclimatation québécoise) mériterait d'être décortiqué d'un point de vue périphérique. 
16

Il y a une dizaine d'années, sous une neige qui se transformait dans un ineffable état de slutch sur la rue Milton, près de l'université McGill, j'ai acheté de seconde main un livre du sociologue américain Robert $\mathrm{K}$. Merton, On the Shoulders of Giants. A Shandean Postscript (New York, Free Press, 1965). Livre curieux qui m'a envoûté au fil des ans. Vaste réflexion/parodie digressive sur les rapports entre fondements culturels et conditions de la découverte en science ou ailleurs. A partir d'un article écrit par lui-même 20 ans auparavant et faussement intitulé "A Note on Science and Democracy ", une enquête se mène rondement sur la fortune de l'aphorisme faussement attribué à Newton «If I have seen farther, it is by standing on the shoulders of giants ». Voyages et acrobaties vont du médiéval Bernard de Chartres à Freud en passant par Burton, Mersenne, C. Bernard, Engels, Peirce et tout l'univers grouillant des érudits. Pensant à cela aujourd'hui dans le kaléidoscope des années 1980, cherchant à comprendre le pitoyable état des bibliothèques académiques francophones et le marché endo-colonial du livre de philosophie. Riant de tous les éclats avec une citation de Ma'me Lalonde «Je suis de la nation renvoyée à une note en bas de page", mise en titre par Jean Métellus dans la Quinzaine littéraire ( ${ }^{\circ} 315$, décembre 1979), à l'époque québécoise de Change et de Jean-Pierre Faye qui allait fonder le Collège international de philosophie avec feu Châtelet et Derrida. Repensant à la belle note dialoguale de A.B. McKillop à propos du livre de Goetzman, The American Hegelians...: "a salutary reminder to students of Canadian thought that significant intellectuals movements need not necessarily emanate from centres of empire " ${ }^{7}$. Me rappellant d'avoir déjà joué du ciseau, exhumant d'un point de vue nord-américain trois ouvrages "nouveaux", respectivement de Michel de Certeau, s.j., Ian Hacking et Antoine Berman; établissant les concordances intra-textuelles pour chacun, remplissant des cahiers de notes à partir ici d'une introduction à caractère autobiographique, là d'une épigraphe de Nietzsche, là d'une note infrapaginale sur la diglossie ${ }^{8}$; fignolant un livre où

7 A. B. McKillop, A disciplined Intelligence. Critical Inquiry and Canadian Thought in the Victorian Era, Montréal, McGill-Queen's Univ. Press, 1979, "Note bibliographique $»$, p. 277.

8 II s'agit dans l'ordre indicatif de La fable mystique. XVI'-XVIP siècle, Paris, Gallimard, 1982; Representing and Intervening. Introductory Topics in the Philosophy of Natural Science, Cambridge, Cambridge Univ. Press, 1983; 
jaurais voulu démontrer l'art total de la recension, une herméneutique "québécoise» dans le stylodrome de la pensée pure. La mort de l'un m'a fait prendre conscience de la folie de l'entreprise; me forcant à voir que chaque auteur a droit à son propre palimpseste, a droit au rewriting moderne de ses premières piétés. Cherchant aujourd'hui à décoder la curieuse voix mélancolique de Jacques Brault: «Les poètes pullulent et les philosophes brillent par leur rareté. Nous ne sommes pas brouillés avec la pensée spéculative, nous en sommes encore à nous éprouver dans la sensation..." ${ }^{9}$ alors qu'il y a a une trentaine d'années, c'est par une modeste «Note sur le langage philosophique» (Dialogue, I, 1962) que la jonction créatrice avait été par lui pressentie.

La note infrapaginale contient le secret ob/scène des rapports entre tradition et création, texture du savoir et blanchissement par ce qui surgit au nom de la science. Â bien des égards, tout se joue sur le cadastre pointilleux de chaque texte, visible ou invisible; la pratique de la note humanise les rapports divers au savoir alors que l'extatique de la non-note attise souverainement une autre pensée "contemporaine". La pensée du Livre de philosophie en tant que livre se constituera ici le jour où intellectuels, philosophes, historiens des faits et des idées transatlantiques, archivistes, critiques littéraires, écrivains diront leur passion de la recherche et du travail, non pas original mais simplement nouveau étant pédagogique, se sentiront responsables du passé de chaque auteur et de l'avenir de chaque lecteur. Pour une déontologie de la pensée pure, voilà mon titre: archivisme poétique de la note qui ferait sauter la pensée par-dessus le filet séparateur, sur le même blanc attentif d'une même page au point de commettre là un texte, un livre souverain, sans plus aucune note, mais qui montrerait à l'évidence le processus ironique,

L'épreuve de l'étranger. Culture et traduction dans l'Allemagne romantique, Paris, Gallimard, 1984. Pendant trois ans, j'ai travaillé de front les virages stylistiques de ce triplet (religion, science, littérature): annoter-recenser un livre, c'est le recréer à partir du zéro à zéro entre milieux de l'auteur et du lecteur.

9 Poussière du chemin, coll. "Papiers collés ", Montreal, Boréal, 1989, p. 15. II est interessant de remarquer que Chemin faisant (Montreal, La Presse, 1975) utilise des commentaires en manchette, que Brault se présente sur la quatrième page de couverture comme a un philosophe à l'état sauvage: plus pensif que penseur" mais que cette collection d'essais ne comporte pas le fameux texte "Pour une philosophie québécoise" (1965). Cette note étant terminée, Brault vient de faire paraître /l n'y a p/us de chemin, Saint-Lambert, Noroît, 1990; à la fois dépouillé et agrippé à plusieurs citations, sur beaucoup de blanc. 
18

marginal ou infrapaginal de sa manifestation ${ }^{10}$. Formes réflexives baptisant de feu leur infra-contenu émergé malgré le roman-fleuve des autorités. Prose qui ne contraindrait plus à mimer la Vérité, à élire les signatures de la "bonne" Philosophie, ni Poème, ni Théorème aveuglant, mais qui ferait accourir chaque lecteur et lectrice VERS LA LIBRE NÉCESSITÉ DE SES PROPRES PENSEES. Si cette question est fondamentale dans notre manière de comprendre l'histoire de la pensée western, elle est ici éclatante, décisive, musculaire. Dans cette province de Québec, partie intégrante et enclavée de la «communauté " atlantique, faire de la philosophie, c'est nécessairement faire voir avec les moyens du bord. Faire bouger les yeux, la tête, le bras, les mains vers autre chose ailleurs, pour ensuite laisser tomber le livre, les mains libres. Comme dirait James Joyce: "Whip your glosses before you rite." Fouettez vos gloses avant d'écrire, et ne regardez plus la page blanche des simulacres géants qui, depuis trente ans d'une Révolution tranquille, vous nomment et entretiennent le décor de votre note exotique.

10 L'absence «créatrice " de notes peut faire apparaître un problème déontologique, là où les a idées " philosophiques se distribuent comme du beurre de pinottes dans les parcs provinciaux. Histoires d'écureuils ou d'écueils! Prenez à bout de bras un exemplaire de Genèse (1982) de Michel Serres, missionnaire d'un "objet nouveau en philosophie", aujourd'hui membre francophone de l'Académie française. Et hop! Deux cent vingt-deux pages, sans notes (sauf trois auto-références et deux «à paraître "), mais qui furent pourtant des notes de cours parlées à l'Université de Montréal, où n'importe quoi est blanc: Curiace et Ulysse, Montaigne, la putain blanchâtre, tous les fleuves sont blancs, l'argent est blanc, retour à l'idée d'indétermination et donc au multiple/virtuel : « Qui suis-je? Un domino blanc, un joker, à toute valeur. Une pure capacite. " (p. 60) Cette banalité sophistiquée, les super-intellectuels en rêvent et la miment à rebours, je le comprends, mais cela laisse aussi entrevoir que le penseur blanchit ses auditeurs (ou ses collègues montréalais déjà sépulcres) et que l'auteur dévore somptueusement ses lecteurs. Style diurétique, dialogue nul. Je n'ai rien contre le style diurétique protégé par la Charte canadienne des droits et libertés, encore faut-il savoir l'endiguer, pour des raisons essentiellement pédagogiques, historiques et anti-gaga, ou alors travailler la notion ironique de copia à partir de Terence Cave, The Cornucopian Text. Problems of Writing in the French Renaissance, Oxford, Clarendon Press, 1979. Immorale de l'histoire dans l'espace transatlantique: "M'y voilà ! faisons-nous Sauvages!", Chateaubriand, Essai sur les révolutions..., Paris, Ladvocat, 1826, t. II, ch. LVI, note infrapaginale. Incarnons réellement la "noise" conceptuelle, la "différance", le "rhizome ", les « petits récits", - nuages philosophiques dans une goutte de leur grammaire -, logeons dans l'élément contradictoire qui fait être leur œuvre grande, abstraite, expansive, mais qui n'a rien de très marginal alors qu'ils ont joue ou font jouer leur manchette. 